\title{
Monocyte adhesion to cerebromicrovascular endothelial cells derived from hypertensive and normotensive rats
}

\author{
R. M. MCCARRON, L. WANG, A.-L. SIRÉN, M. SPATZ, AND J. M. HALLENBECK \\ Stroke Branch, National Institute of Neurological Disorders and Stroke, Bethesda, Maryland 20892
}

\begin{abstract}
McCarron, R. M., L. Wang, A.-L. Sirén, M. Spatz, and J. M. Hallenbeck. Monocyte adhesion to cerebromicrovascular endothelial cells derived from hypertensive and normoten. sive rats. Am. J. Physiol. 267 (Heart Circ. Physiol. 36): H2491-H2497, 1994.-The stroke risk factor hypertension may function as a predisposing agent by increasing the vulnerability of blood vessels to thrombosis or hemorrhage. The research here demonstrates that cerebrovascular endothelial cells (EC) from spontaneously hypertensive (SHR) and Wistar-Kyoto normotensive (WKY) rats exhibit similar levels of adhesiveness for syngeneic peripheral blood monocytes (e.g. $22.53 \pm 1.32$ and $24.35 \pm 1.16 \%$, respectively). Monocyte adhesion to SHR EC was dramatically increased by treatment of EC with lipopolysaccharide, interferon- $\gamma$, or interleukin- $1 \beta$ and tumor necrosis factor- $\alpha$ (e.g., 106,68 , and $171 \%$, respec. tively). Identical treatment of WKY EC also increased adhesion albeit at significantly lower levels than observed on concomitantly tested SHR EC (e.g., 47.8, 12.7, and 60.7\%, respectively). Allogeneic combinations of monocytes and EC again demonstrated significantly more upregulation of adhesion by treatment of SHR EC than WKY EC. Characterization of these adhesive interactions revealed the interplay of adhesion pathways, which include lymphocyte functional antigen1/intercellular adhesion molecule-1 (ICAM-1), Mac-1/ICAM-1, and very late activation antigen-4/vascular adhesion molecule-1 as well as other undetermined mechanisms. In summary, these findings indicate hypertension may enhance responsiveness of endothelium to factors that promote monocyte adhesion.
\end{abstract}

intercellular adhesion molecule-1; vascular adhesion molecule-1; tumor necrosis factor- $\alpha$; interleukin-1 $\beta$; lipopolysaccharide

ADHESIVE INTERACTIONS involving peripheral blood monocytes and vascular endothelium are important and early events under normal (steady-state) conditions as well as in response to inflammation or vascular injury $(4,31)$. These adhesive interactions are mediated by surface molecules expressed by monocytes and endothelial cells (EC). These include lymphocyte functional antigen-1 (LFA-1) (CD11a/CD18), Mac-1 (CD11b/CD18), p150,95 (CD11c/CD18), very late activation antigen-4 (VLA-4) (CD49d/CD29), and sialyl Lewis ${ }^{x}$ molecules expressed by monocytes and intercellular adhesion molecule-1 (ICAM-1) (CD54), ICAM-2, E-selectin, vascular adhesion molecule-1 (VCAM-1), and granule membrane protein-140 (GMP-140) (CD62) expressed by EC $(4,8,23)$. The expression of these molecules by vascular EC can be upregulated in vitro by a variety of factors such as interleukin-1 $\beta$ (IL-1 $\beta$ ), tumor necrosis factor- $\alpha$ (TNF $\alpha$ ), interferon- $\gamma$ (IFN $\gamma$ ), and lipopolysaccharide (LPS) (11, $16,17,22)$. The endothelial expression of molecules such as ICAM-1 and its ability to promote adhesion of peripheral blood monocytes, as well as other leukocytes, has been widely studied $(3,8,11,16)$. Adhesive interac- tions involving vascular endothelium play important roles in a variety of disorders, including autoimmune murine lupus nephritis (33), hemorrhagic shock (20), experimental allergic encephalomyelitis $(12,16)$, multiple sclerosis (28), and cerebral ischemic stroke (9). In particular, the role of monocyte-EC interactions in the pathogenesis of atherosclerosis has been widely studied $(10,14,24)$.

ICAM-1 expression by cultured cerebromicrovascular EC derived from spontaneously hypertensive rats (SHR) was shown to be more sensitive to cytokine-induced upregulation than similar cultures derived from normotensive Wistar-Kyoto (WKY) and Sprague-Dawley (SD) rats (17). These findings may indicate a mechanism at least partially responsible for increases in in vivo perivascular monocyte accumulation seen in hypertensive rats $(15,27)$. Some of these effects may be mediated by the production of proinflammatory factors such as $\mathrm{TNF}_{\alpha}$, which is produced in greater amounts by SHR than by WKY rats when injected with LPS $(26,27)$. These observations indicate a possible mechanism by which hypertension may predispose to the development of disorders such as atherosclerosis as well as stroke. Hypertension may be a risk factor by virtue of its ability to affect responsiveness of cerebrovascular EC to factors regulating interactions with monocytes. It is known that adhesion molecules other than just LFA-1/ICAM-1 or Mac-1/ICAM-1 (i.e., VLA-4/VCAM-1) are important in monocyte-EC interactions $(3,6,19)$. The availability of cultured cerebrovascular EC allows for the determination of the relative importance of these pathways in the abovementioned cell interactions. In addition, the concomitant study of EC derived from normotensive and hypertensive rats permits direct comparison of their capacity to respond to various stimuli.

\section{METHODS}

Rats. Female WKY and SHR rats obtained from Taconic Farms (Germantown, NY) and SD rats obtained from Harlan Sprague Dawley (Indianapolis, IN) were used at 12-14 wk of age to isolate cerebromicrovascular EC and peripheral blood monocytes. All animal procedures were performed in accordance with the NIH "Guide for the Care and Use of Laboratory Animals" [Department of Health and Human Services (NIH) Publication No. 86-23, Revised 1985].

Cerebromicrovascular EC cultures. The isolation and culti. vation of cerebromicrovascular EC from SHR, WKY, and SD rats were performed with some modification as previously described (29). All EC cultures routinely exhibited characteristic cobblestone appearances and were used at the time they attained confluence on 96-well flat-bottom microtiter plates. Cultures contained $>95 \%$ EC identified by immunocytochemistry and fluorescence-activated cell sorter (FACS) analysis using EC-specific anti-factor VIII-related antigen (Accurate Chemical and Scientific, Westbury, NY). EC cultures used in experiments described here were obtained from three separate 
lines ( 1 line for SD EC) and were used at third through seventh passages.

Before use in adhesion assays, EC monolayers were washed twice with warm Hanks' balanced saline solution (HBSS) followed by incubation in media alone or with indicated concentrations of recombinant IFN $\gamma$ (Genzyme, Boston, MA), human IL-1 $\beta$ (Endogen; Boston, MA), recombinant human TNF $\alpha$ (Endogen), or lipopolysacharide (LPS, Sigma, St. Louis, MO). The media consisted of medium 199 (M-199) (containing $25 \mathrm{mM} N$-2-hydroxyethylpiperazine- $N^{\prime}$-2-ethanesulfonic acid buffer, Earle's salts, and L-glutamine) with $20 \%$ heatinactivated fetal calf serum (FCS), $90 \mu \mathrm{g} / \mathrm{ml}$ heparin, 100 $\mu \mathrm{g} / \mathrm{ml}$ streptomycin, $100 \mathrm{U} / \mathrm{ml}$ penicillin $\mathrm{G}$, and $0.25 \mu \mathrm{g} / \mathrm{ml}$ amphotericin B as fungizone (all from GIBCO, Long Island, $\mathrm{NY}$ ), and $20 \mu \mathrm{g} / \mathrm{ml}$ endothelial cell growth supplement (Collaborative Research; Bedford, MA). All cytokines contained $<10$ $\mathrm{pg} / \mathrm{ml}$ endotoxin as determined by E-toxate assay kit (Sigma). ICAM-1 expression by EC cultures was quantitated by enzymelinked immunosorbent assay as previously described (30).

Monocytes. Peripheral blood was obtained from rats by cardiac puncture and collected into heparin-coated tubes. Mononuclear cells were isolated by Ficoll-Hypaque density gradient centrifugation (Histopaque 1119/Histopaque 1077; Sigma) for $30 \mathrm{~min}$ at $700 \mathrm{~g}, 24^{\circ} \mathrm{C}$. Mononuclear cells separated from plasma, and granulocytes were collected, washed, and further purified by centrifugation on hyperosmotic Nycodenz density gradients (Accurate Chemical, Westbury, NY) for 15 min at $600 \mathrm{~g}, 24^{\circ} \mathrm{C}$. Monocyte preparations were extensively washed in PBS containing $0.5 \%$ bovine serum albumin (BSA) and $2 \%$ EDTA to remove contaminating platelets. The monocyte-enriched fractions were counted ( $>95 \%$ viable as determined by trypan blue exclusion), and their purity was assessed as $>90 \%$ by their ability to phagocytize latex beads and by FACS analysis using an antibody to rat monocytes and macrophages (ED-1) (see Table 1).

Monocyte adhesion. Adhesion experiments were performed using monocytes labeled with sodium $\left[{ }^{51} \mathrm{Cr}\right]$ chromate $(0.1$ $\mathrm{mCi} / \mathrm{ml}, 30 \mathrm{~min}, 37^{\circ} \mathrm{C}$ ). Cells were washed twice and resuspended (usually $4-10 \times 10^{5} / \mathrm{ml}$ ) in M-199. Before the addition of monocytes, EC cultures were washed twice with warm HBSS containing 4\% FCS. Monocytes were added ( $50 \mu \mathrm{l} /$ well), and plates were incubated at $37^{\circ} \mathrm{C}$ for $30 \mathrm{~min}$. After incubation, nonadherent cells were removed, and monolayers were gently washed twice with warm M-199. The adherent cells were lysed by overnight incubation with $2 \%$ (vol/vol) Triton-X $(0.2 \mathrm{ml} /$ well $)$, and radioactivity in all fractions was counted on

Table 1. Expression of adhesion molecules by SHR, WKY, and SD monocyte populations

\begin{tabular}{llll}
\hline \hline \multirow{2}{*}{ MAb } & \multicolumn{3}{c}{ Positively Stained Cells, \% } \\
\cline { 2 - 4 } & \multicolumn{1}{c}{ SHR Mo } & \multicolumn{1}{c}{ WKY Mo } & SD Mo \\
\hline ED-1 & $90.3(142.3)$ & $89.6(129.3)$ & $92.7(123.6)$ \\
ED-2 & $11.3(19.60)$ & $8.7(19.7)$ & $7.9(23.2)$ \\
CD11a & $81.4(168.3)$ & $77.6(156.9)$ & $68.4(160.0)$ \\
CD11b & $\mathbf{8 4 . 4 ( 1 5 1 . 7 )}$ & $86.0(141.8)$ & $70.6(124.4)$ \\
CD18 & $79.6(237.4)$ & $86.5(183.7)$ & $72.4(133.7)$ \\
CD54 & $32.0(108.4)$ & $41.5(123.5)$ & $40.6(159.5)$ \\
VLA-4 & $28.2(31.6)$ & $22.4(25.9)$ & $23.3(27.0)$ \\
\hline
\end{tabular}

Expression of indicated markers on freshly prepared peripheral blood monocytes (Mo) were analyzed by flow cytometry. Data from one representative experiment are presented as percentage of positively stained cells with mean fluorescence intensity values in parentheses as described in METHODS. Freshly prepared Mo were incubated with indicated monoclonal antibodies (MAb) as described in METHODS. SHR, spontaneously hypertensive rats; WKY, Wistar-Kyoto rats; SD Sprague-Dawley rats. VLA-4, very late activation antigen-4 a LKB Compugamma 1282 gamma counter. The percentage of adherent cells was calculated by dividing Triton-X counts per minute by the total counts per minute added. All experiments were performed in quadruplicate.

Because monocyte preparations were partially contaminated with lymphocytes, which also adhere to endothelium, some experiments calculated adhesion by measuring myeloperoxidase (MPO) activity (2), which is present in monocytes and granulocytes, but not lymphocytes. No significant variations in the percent adhesion were observed using radioactivity data and data assessing MPO activity. Also, ED-1 staining of EC monolayers after addition of monocytes showed that $<10 \%$ of the adhering cells were unstained, indicating that adhesion by contaminating lymphocytes was not a factor under the conditions used here.

Antibody-blocking experiments. Before addition to EC monolayers, monocytes were preincubated in serum-free media for $20 \mathrm{~min}$ at $37^{\circ} \mathrm{C}$ with saturating concentrations $(10-30 \mu \mathrm{g} / \mathrm{ml})$ of indicated monoclonal antibodies (MAbs) or in serum-free medium alone. Isotype-matched antibodies were used as controls and found to have no significant effects compared with untreated monocytes. In blocking experiments using antiICAM-1(1A29), EC monolayers were preincubated with an antibody for $30 \mathrm{~min}$ and washed three times with warm HBSS containing 4\% FCS before addition of monocytes. Adherence was determined as described above, and inhibition was calculated by the formula: $100 \% \times$ [\%adhesion (no MAb) \%adhesion (+ MAb)]/\%adhesion (no MAb).

Flow cytometry. Analysis of cell surface molecule expression on peripheral blood monocytes freshly prepared from both SHR and WKY rats was performed by staining with antibodies as described below (1:200-1:500 dilution; except clone WT.5, which was used at $1: 50$ ) for $0.5-1 \mathrm{~h}$ at $4^{\circ} \mathrm{C}$. After being washed (PBS containing $0.02 \mathrm{mM}$ sodium azide, 1\% BSA) and incubated $\left(30 \mathrm{~min}, 4^{\circ} \mathrm{C}\right)$ with 1:50 rhodamine-conjugated goat $\mathrm{F}\left(\mathrm{ab}^{\prime}\right)_{2}$ anti-mouse immunoglobulin (Ig)G (Accurate), cells were washed and analyzed by flow cytometry using a FACS IV analyzer (Becton Dickinson FACS System, Mountain View, CA). The data are presented as percentage of cell population positively stained and mean fluorescence intensity (MFI) representing semiquantitative data (in channel units) on the mean number of fluorescent reagent-binding molecules expressed by the cells.

Antibodies. Anti-rat ICAM-1 (CD54; clone 1A29), anti-rat VLA-4 (CD49d; clone TA-2), anti-rat LFA-1 $\alpha$ chain (CD11a; clone WT.1), and anti-rat LFA-1 $\beta$ chain (CD18; clone WT.3) are mouse IgG1; anti-rat Mac- $1 \alpha$ chain (CD11b; clone WT.5) is a mouse IgA. All antibodies were obtained from Seikagaku America (Rockville, MD). Monoclonal anti-rat ED-1 and antirat ED-2 are both mouse IgG $_{1}$ antibodies obtained from Biosource International (Camarillo, CA).

\section{RESULTS}

Purified populations of unstimulated monocytes from SHR, WKY, and SD rats were $>90 \%$ monocytes as judged by their expression of ED-1 antigen, which is present on monocytes and macrophages but not granulocytes (Table 1 ). Only a small percentage of cells stained positively for ED-2, which is expressed by macrophages but not monocytes or granulocytes. The monocyte populations from all three rat strains also contained many cells positively stained for adhesion molecules CD11a (LF-1 $\alpha$ ), CD11b (Mac-1 $\alpha$ ), and CD18 (LFA-1 $\beta$ ). A somewhat smaller percentage of cells stained positively for ICAM-1 (CD54) and even fewer for VLA-4 (CD49d). In experiments performed on other monocyte preparations 
from the same rat strains, the relative degree of expression of the listed markers was similar except for the expression of VLA-4 and ICAM-1, which fluctuated by as much as $20 \%$.

The adherence of peripheral blood monocytes freshly isolated from SHR and WKY rats was determined using syngeneic EC monolayers. In these experiments, the number of monocytes that adhered to EC monolayers was directly proportional to the number of monocytes added (Fig. 1). The percentage of adherent monocytes within a given population was constant over a wide range of concentrations (1-7.5 $\times 10^{4}$ monocytes/ microtiter well). The use of $>8 \times 10^{4}$ monocytes yielded significant changes in the percentage of adherent monocytes, which were probably due in part to overcrowding and to the adherence of monocytes to monocytes already adhering to EC monolayers (observed by light microscopy). In different experiments, the percentage of monocytes that adhered to unstimulated EC monolayers varied only slightly (Fig. 1). These variations were dependent on the EC culture (i.e., passage number) and the monocyte preparation.

In a representative experiment (Fig. 2), there were no significant differences in the binding levels of peripheral blood monocytes freshly isolated from SHR and WKY rats to syngeneic monolayers of unstimulated EC (i.e., $22.73 \pm 1.42$ and $25.33 \% \pm 1.26$, respectively). In four separate experiments, which used EC cultures established and passaged simultaneously and monocytes isolated from age-matched and identically treated rats, no significant differences were observed in the basal level of

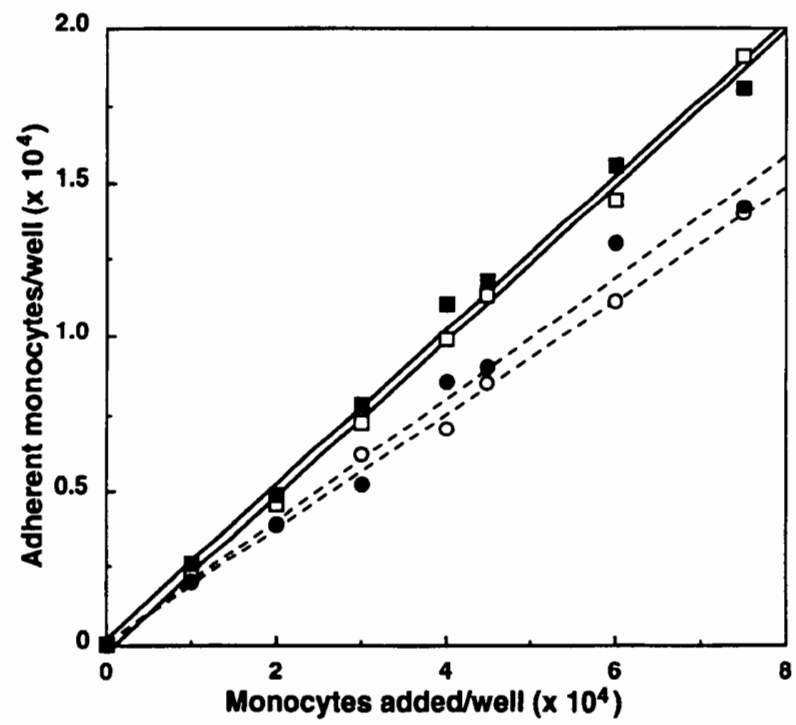

Fig. 1. Effect of number of monocytes added on number of monocytes adhering to unstimulated syngeneic endothelial cell (EC) monolayers. Monocytes freshly prepared from peripheral blood of spontaneously hypertensive rats (SHR, $\square, 0$ ) and Wistar-Kyoto (WKY, ם, ๑) rats were added to syngeneic EC monolayers. Results are from 2 separate experiments (expt 1, solid lines; expt 2, dashed lines). Each point represents mean of quadruplicate wells (all means $\pm \mathrm{SE}$ values were within $10 \%$ of the mean). Numbers of monocytes were determined by evaluation of standards that measured ratio of counts per minute of $\mathrm{Cr}$ per cell counts. Estimates by microscopic evaluation of ED-1 positive cells were similar to determinations by radioactive counting.

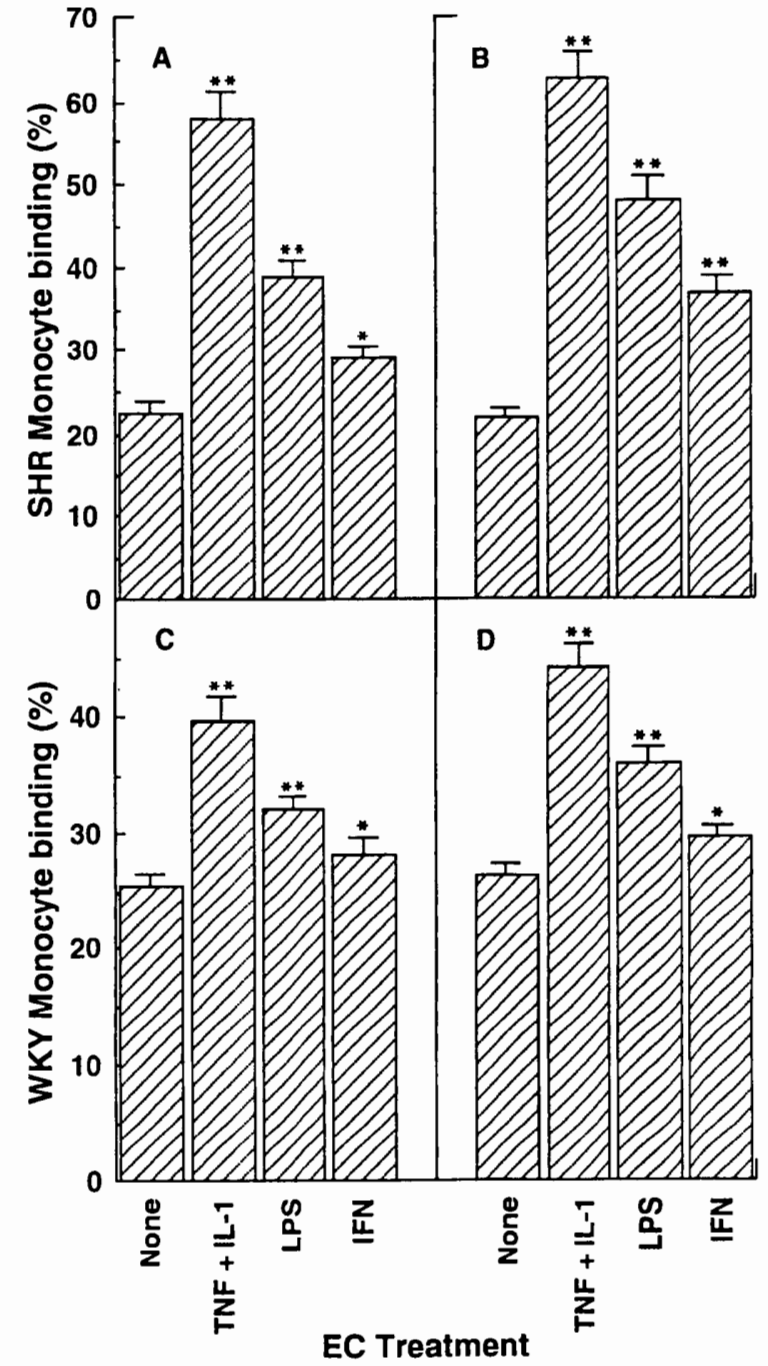

Fig. 2. Effect of EC stimulation on SHR and WKY monocyte binding to syngeneic EC. EC monolayers derived from SHR $(A$ and $B)$ and WKY ( $C$ and $D$ ) rat cerebral microvessels were treated for $4 \mathrm{~h}$ ( $A$ and $C$ ) or $20 \mathrm{~h}(B$ and $D$ ) with $100 \mathrm{U} / \mathrm{ml}$ interferon (IFN), $100 \mathrm{ng} / \mathrm{ml}$ lipopolysaccharide (LPS), or $100 \mathrm{U} / \mathrm{ml}$ tumor necrosis factor (TNF) + $40 \mathrm{U} / \mathrm{ml} \mathrm{IL}-1$. Data are means $\pm \mathrm{SE}$ of quadruplicate wells obtained from a single representative experiment $\left({ }^{*} P=<0.01,{ }^{* *} P=<0.001\right.$; Student's $t$-test).

SHR or WKY monocyte adhesion to untreated EC monolayers.

The treatment of EC cultures with LPS, IFN $\gamma$, or TNF $\alpha+\mathrm{IL}-1 \beta$ for 4 or $20 \mathrm{~h}$ increased their adhesiveness for monocytes (Fig. 2). The increase in SHR monocyte adhesion to TNF $\alpha+$ IL-1 $\beta$-treated SHR EC ( $>150 \%)$ was significantly greater $(P<0.001)$ than that observed with WKY monocytes to similarly treated WKY EC (55\%). This greater responsiveness of SHR EC was observed for all treatments and was significant at both 4- and 20-h time periods. Similar results were also observed in experiments using other monocyte preparations and EC cultures.

To assess whether this difference is attributable to monocytes or EC, a direct comparison of the adhesion of SHR and WKY monocytes was performed by simultaneously assessing adhesion to syngeneic as well as 
allogeneic EC monolayers. The results demonstrate that both monocyte populations exhibited increased adhesiveness to stimulated ( $20 \mathrm{~h}$ ) allogeneic EC monolayers (Fig. 3 ). The increased adhesiveness followed the same pattern as was seen in the syngeneic combinations (i.e., TNF $\alpha+$ IL-1 $\beta>$ LPS > untreated). The relative increase in adhesion of either monocyte population to stimulated SHR EC was significantly greater than was observed with WKY EC $(P<0.001)$. Similar results were also observed using EC treated for $4 \mathrm{~h}$ and in identical experiments performed using different monocyte preparations and EC lines (results not shown).

Peripheral blood monocytes freshly isolated from SD rats also adhered to a significantly greater degree to treated SHR EC than to treated WKY or SD EC (Table 2). The quantitative level of upregulation of SD EC adhesiveness by $\mathrm{TNF} \alpha+\mathrm{IL}-1 \beta$ was similar to that observed with WKY EC. In all cases, the relative increases in adhesion as a result of EC treatment were significantly greater for SHR EC than for WKY or SD

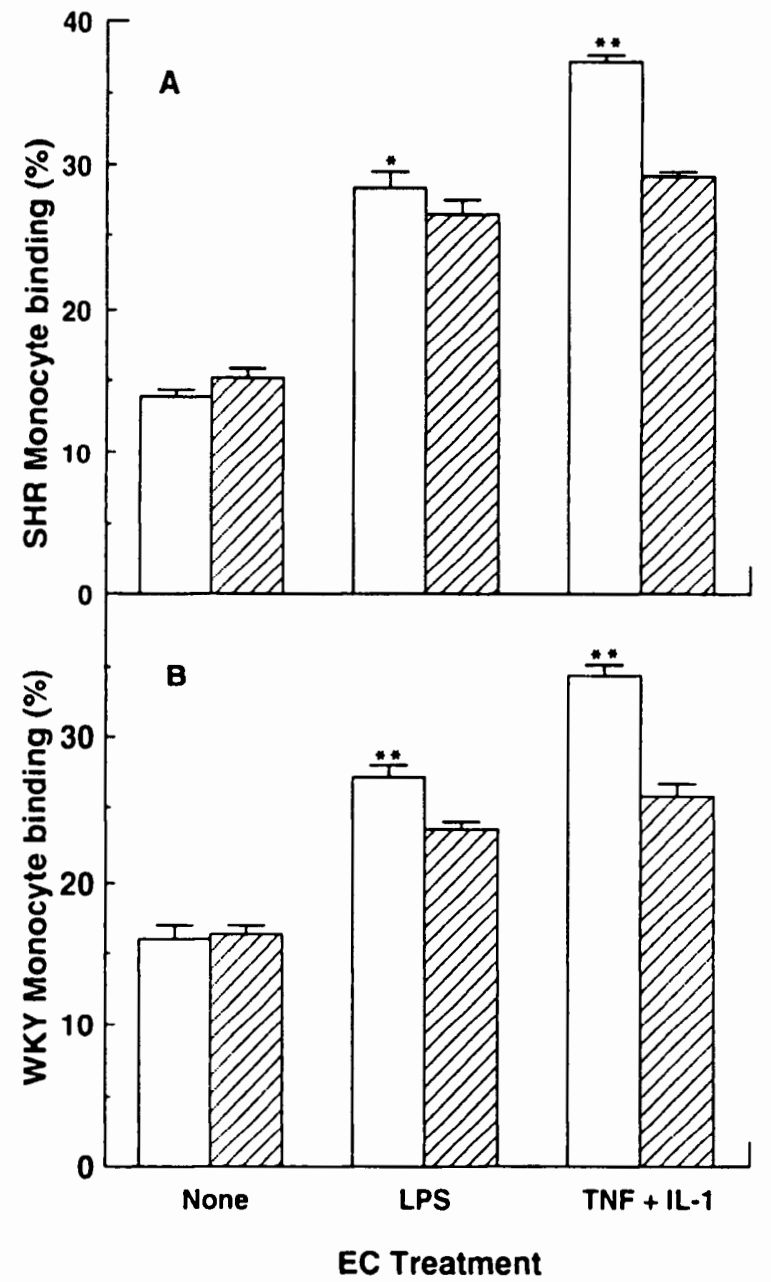

Fig. 3. Effect of EC stimulation on SHR and WKY monocyte binding to allogeneic and syngeneic EC. Freshly prepared peripheral blood monocytes from SHR $(A)$ and WKY $(B)$ rats were concomitantly allowed to adhere to EC monolayers derived from SHR (open bars) and WKY (hatched bars) rat cerebral microvessels treated for $20 \mathrm{~h}$ with $100 \mathrm{ng} / \mathrm{ml} \mathrm{LPS}$ or $100 \mathrm{U} / \mathrm{ml} \mathrm{TNF}+40 \mathrm{U} / \mathrm{ml} \mathrm{IL}-1$. Data are means \pm $\mathrm{SE}$ of quadruplicate wells obtained from a single representative experiment $\left({ }^{*} P=<0.01,{ }^{* *} P=<0.001\right.$; Student's $t$-test $)$.
Table 2. Enhancement of monocyte adhesion to stimulated SHR, WKY, and SD cerebrovascular EC cultures

\begin{tabular}{lccc}
\hline \hline \multirow{2}{*}{ EC Treatment } & \multicolumn{3}{c}{ Increased Adhesion, \% } \\
\cline { 2 - 4 } & SHR EC & WKY EC & SD EC \\
\hline & \multicolumn{3}{c}{ SHR monocytes } \\
LPS & 106.3 & $72.8^{*}$ & $66.7^{*}$ \\
TNF + IL-1 & 170.5 & $117.2^{*}$ & $109.6^{*}$ \\
IFN & 68.0 & $34.8^{*}$ & $\mathrm{ND}$ \\
& & WKY monocytes \\
LPS & 76.1 & $47.8^{*}$ & $41.9^{*}$ \\
TNF + IL-1 & 93.2 & $60.7^{*}$ & $54.0^{*}$ \\
IFN & 14.6 & $12.7^{-6}$ & $\mathrm{ND}$ \\
& & $S D$ monocytes \\
LPS & 80.3 & $40.9^{*}$ & $51.3^{*}$ \\
TNF + IL-1 & 89.0 & $54.9^{*}$ & $69.1^{*}$ \\
\hline
\end{tabular}

Data from 3 separate experiments were pooled and are presented as percentage of increased adhesion according to the formula: $100 \% \times[\%$ adhesion (test) - $\%$ adhesion (control) $) / \%$ adhesion (control). Control values for adhesion of SHR, WKY, and SD monocytes to unstimulated endothelial cells (EC) were not significantly different and ranged between 14.83 and $23.96 \%$. Cerebrovascular EC cultures were incubated for $20 \mathrm{~h}$ with indicated factors at the following concentrations: $100 \mathrm{ng} / \mathrm{ml}$ lipopolysaccaride (LPS); $100 \mathrm{U} / \mathrm{ml}$ tumor necrosis factor (TNF) + $40 \mathrm{U} / \mathrm{ml}$ interleukin-1 (IL-1); or $100 \mathrm{U} / \mathrm{ml}$ interferon (IFN). * Significant (2-way analysis of variance and Bonferroni correction; $P$ $<0.001$ ) differences between SHR EC and WKY or SD EC in the levels of upregulated monocyte adhesion for each respective EC culture. ND, not done.

EC (2-way analysis of variance and Bonferroni correction; $P<0.001$ ).

The adhesion molecules responsible for the observed monocyte-EC interactions were studied by antibodyblocking experiments. The adherence of WKY monocytes to unstimulated WKY EC monolayers was partially inhibited (10.7 and $42.7 \%$ ) by incubation of monocytes with MAb against $\alpha$-chains of LFA-1 or Mac-1, respectively (Table 3 ). Incubation with antibodies to the common $\beta$-chain (CD18) of these two adhesion molecules inhibited adhesion by $>50 \%$. An antibody

Table 3. Effect of anti-adhesion antibody treatment on WKY monocyte adhesion to syngeneic cerebrovascular EC cultures

\begin{tabular}{llll}
\hline \multirow{2}{*}{$\begin{array}{c}\text { Antibody } \\
\text { Treatment }\end{array}$} & \multicolumn{3}{c}{ EC Treatment } \\
\cline { 2 - 4 } & \multicolumn{1}{c}{ None } & TNF + IL-1 (4 h) & TNF + IL-1 (20 h) \\
\hline None & 22.5 & 33.6 & 38.5 \\
CD18 & $10.2(54.7)$ & $27.1(19.4)$ & $34.6(10.1)$ \\
CD1 la & $20.1(10.7)$ & $31.4(6.6)$ & $36.4(5.5)$ \\
CD11b & $12.9(42.7)$ & $27.6(17.9)$ & $33.3(13.5)$ \\
VLA-4 & $23.1(0)$ & $30.9(8.1)$ & $33.8(12.2)$ \\
CD18/VLA-4 & $11.3(49.8)$ & $22.0(34.5)$ & $21.9(49.4)$ \\
ICAM-1 & $11.1(50.7)$ & $26.3(21.7)$ & $33.7(12.1)$ \\
\hline
\end{tabular}

Data are presented as percent adhesion calculated as described in METHODS. Cerebrovascular EC culture monolayers were incubated for 4 or $20 \mathrm{~h}$ in the absence or presence of $100 \mathrm{U} / \mathrm{ml} \mathrm{TNF}+40 \mathrm{U} / \mathrm{ml} \mathrm{IL}-1$. Numbers in parentheses represent percent inhibition, which was calculated according to the formula: $100 \% \times$ [\%adhesion (no MAb) - \%adhesion (+MAb)]/\%adhesion (no MAb). Monocytes or EC were incubated with indicated MAbs as described in METHODS. ICAM-1, intercellular adhesion molecule- 1 . 
directed against rat VLA-4 had no effect on adhesion to unstimulated EC monolayers. The combination of antibodies to CD18 and VLA-4 did not further enhance the inhibition of monocyte adhesion that was observed by treatment with anti-CD18 alone (Table 3). The capacity of antibody to ICAM-1 to block monocyte adhesion to untreated EC monolayers was equal to the inhibition observed with antibodies to its ligands (CD11a/CD18 and CD11b/CD18), which are present on the monocytes. The findings also indicate that an additional ICAM-1 ligand (i.e., p150,95 $\alpha$-chain; CD11c) probably does not play a major role in monocyte adhesion to nonstimulated EC.

The treatment of monocytes with anti-adhesion molecule antibodies inhibited adhesion to cytokine-treated EC to varying degrees. The ability to block adhesion mediated by LFA-1/ICAM-1 or Mac-1/ICAM-1 significantly dropped with increasing time of incubation (i.e., anti-CD18 inhibited adhesion $54.7,19.4$, and $10.1 \%$ to EC monolayer untreated or treated with TNF $\alpha+$ IL-1 $\beta$ for 4 and $20 \mathrm{~h}$, respectively). Similar findings were observed with treatment of EC monolayers with antiICAM-1. In addition, the adhesion of monocytes to treated EC monolayers became increasingly sensitive to inhibition by antibody to VLA-4. Finally, the combination of anti-CD18 and anti-VLA-4 was significantly less effective at inhibiting monocyte adhesion to EC treated for $4 \mathrm{~h}$ with TNF $\alpha+\mathrm{IL}-1 \beta$ (34.5\%) than to EC treated for $20 \mathrm{~h}$ or untreated (49.4 and $49.8 \%$, respectively). Very similar levels of inhibition by antibody treatment were observed in experiments using different WKY EC cultures.

An almost identical pattern of inhibition by antibodies was observed using SHR monocytes and SHR EC monolayers (Table 4). Although the degree of upregulation of adhesion with TNF $\alpha+$ IL-1 $\beta$ was considerably greater in these cultures than in the WKY cultures shown in Table 2 (i.e., 87.1 vs. $49.3 \%$ at $4 \mathrm{~h}$ and 120.7 vs. $71.1 \%$ at $20 \mathrm{~h}$, respectively), there were no significant differences in the relative capacity of antibodies to inhibit adhesion. The data shown in Table 4 are representative of experiments performed on three different SHR EC cultures.

Table 4. Effect of anti-adhesion antibody treatment on SHR monocyte adhesion to syngeneic cerebrovascular EC cultures

\begin{tabular}{llll}
\hline \multirow{2}{*}{$\begin{array}{c}\text { Antibody } \\
\text { Treatment }\end{array}$} & \multicolumn{3}{c}{ EC Treatment } \\
\cline { 2 - 4 } & \multicolumn{1}{c}{ None } & TNF + IL-1 (4 h) & TNF + IL-1 (20 h) \\
\hline None & 21.7 & 40.6 & 47.9 \\
CD18 & $11.6(46.5)$ & $32.0(21.2)$ & $42.6(11.1)$ \\
CD11a & $18.9(12.9)$ & $38.1(6.2)$ & $45.9(4.2)$ \\
CD11b & $13.0(40.1)$ & $31.6(22.2)$ & $40.8(14.8)$ \\
VLA-4 & $21.9(0)$ & $36.4(10.3)$ & $40.1(16.3)$ \\
CD18/VLA-4 & $11.3(47.9)$ & $26.5(34.7)$ & $25.9(45.9)$ \\
ICAM-1 & $10.5(51.6)$ & $31.3(22.9)$ & $40.9(14.6)$ \\
\hline
\end{tabular}

Data are presented as percent adhesion calculated as described in METHODS. Cerebrovascular EC culture monolayers were incubated for $4 \mathrm{~h}$ or $20 \mathrm{~h}$ in absence or presence of $100 \mathrm{U} / \mathrm{ml} \mathrm{TNF}+40 \mathrm{U} / \mathrm{ml} \mathrm{IL-1}$. Numbers in parentheses represent percent inhibition, which was calculated according to the formula: $100 \% \times$ [\%adhesion (no MAb) - \%adhesion (+MAb)]/\%adhesion (no MAb). Monocytes or EC were incubated with indicated MAbs as described in METHODS.

\section{DISCUSSION}

The experiments here demonstrate that stimulation of EC monolayers (LPS or TNF $\alpha+$ IL-1 $\beta$ ) considerably increased syngeneic monocyte adhesion to SHR EC to a greater degree than to WKY EC (Fig. 2). Similar results were obtained using allogeneic combinations of monocytes and EC (Fig. 3, Table 2), indicating that these differences are directly related to the responsiveness of the EC cultures.

A possible explanation for these findings involves the expression of adhesion molecules such as ICAM-1. This molecule is known to be expressed by brain EC $(12,16$, $17,28)$ and implicates these cells in interactions with peripheral blood lymphocytes, monocytes, and neutrophils that express CD11a, CD11b, and CD18 integrins $(3,8,11,19)$. The involvement of ICAM-1 and its ligands in monocyte-EC interactions described here was examined by antibody-blocking studies. These experiments may also discern which specific adhesion pathways are responsible for the enhanced adhesiveness of treated SHR EC. The results showed that $\sim 50 \%$ of monocyte adhesion to unstimulated EC monolayers was due to Mac-1 (CD11b/CD18) and, to a lesser extent, LFA-1 (CD11b/CD18) interactions with ICAM-1. Antibodyblocking experiments were also performed on EC treated with TNF $\alpha+$ IL- $1 \beta$ for 4 and $20 \mathrm{~h}$. Although either of these cytokines alone similarly affected EC cultures, maximal responses were obtained with mixtures of both cytokines. In addition, these cytokines were used in combination, since both are concomitantly produced and most often present together. Considerably decreased blocking by treatment with above antibodies was seen on EC treated with TNF $\alpha+$ IL-1 $\beta$. This result indicated either a decrease in the role for these adhesion molecules or, more likely, the emergence of additional adhesion pathways responsible for monocyte-EC interactions. The observation that stimulated EC express even more ICAM-1 (16) strongly suggest the latter.

Antibody-blocking studies also showed that treatment of EC with TNF $\alpha$ + IL-1 $\beta$ resulted in time-dependent increases in sensitivity to blocking by VLA-4 antibody. These data suggest that monocyte interactions with stimulated but not unstimulated EC are partially dependent on VLA-4 expressed by the monocyte and implicate the EC expression of VCAM-1 (a ligand for the integrin VLA-4) in interactions between monocytes and stimulated EC. The expression of VCAM-1 by EC has been described $(6,7,18)$. The observation that VLA-4 is expressed on freshly prepared monocyte preparations (Table 1), and the lack of an effect of VLA-4 antibody on adhesion to unstimulated EC suggest the absence of VCAM-1 expression on unstimulated brain microvessel EC. Little to no VCAM-1 is expressed on unstimulated endothelium $(3,7,19)$. However, its expression by $\mathrm{EC}$ is induced by cytokine treatment $(6,7,18)$, and this implicates stimulated EC in interactions with cells expressing VLA-4, including resting lymphocytes and monocytes but not granulocytes $(6,21)$. A pathogenic role for VCAM-1 in monocyte adhesion is implicated by its expression on endothelium during early atheroscle- 
rotic lesion formation in hypercholesterolemic (dietaryinduced) and Watanabe heritable hyperlipidemic rabbits (heritable models) (10).

Treatment of monocytes with either VLA-4 antibody or CD18 antibody had relatively little effect on adhesion to stimulated EC, whereas the combination of both antibodies inhibited adhesion by almost $50 \%$ (Tables 3 and 4). Similar findings have been reported in studies examining monocyte adhesion to human umbilical vein endothelial cells $(6,19)$ and imply the coincident expression of two operational pathways for monocyte adhesion. The inability of the above two monoclonal antibodies to totally block adherence indicate that other molecules are also involved in the interactions reported here. The in vivo relevance of these findings is shown by experiments demonstrating inhibition of monocyte emigration into inflamed rabbit peritoneum by intravenous infusion of MAb to CD18 and VLA-4 (32). The lack of any effect by treatment with individual MAbs implies that blockade of both pathways is required to suppress extravasation of monocytes. Interestingly, the treatment of monocytes with the combination of antibodies to CD18 and VLA-4 inhibited adhesion to EC treated for $20 \mathrm{~h}$ to a significantly greater extent than to EC similarly treated for $4 \mathrm{~h}$ (Tables 3 and 4). Early and transient expression of other adhesion molecules by stimulated EC may account for this observation. A possible candidate molecule would be E-selectin, which is synthesized de novo by cytokine-treated EC, is transiently expressed on EC $(2-8 \mathrm{~h})$, and interacts with circulating blood cells that express sialyl Lewis ${ }^{\mathrm{X}}$ (i.e., monocytes) $(5,6,18)$.

Although a role for ICAM-1 (and possibly VCAM-1) in cytokine-mediated monocyte adhesion in this system is established, the involvement of these molecules in migration is unknown. It is also not known if these same factors will similarly affect EC derived from differentsized vessels. The most interesting observation described here is that adhesion of any particular monocyte preparation to treated SHR EC is greater than to similarly treated WKY or SD EC (Table 2). This enhanced responsiveness of EC may be due to a factor related to hypertension. More conclusive evidence awaits testing of other hypertensive and normotensive rat strains, as well as experiments utilizing experimentally induced hypertension models and hypertensive strains treated with antihypertensive medications.

It is interesting that all experiments concomitantly performed using SHR and WKY monocytes also indicate some differences in the capacity of monocytes to adhere to stimulated EC. In examining the adhesion of these monocyte populations to syngeneic and allogeneic EC, the adhesion of unstimulated SHR monocytes to treated EC was consistently and significantly more upregulated than that observed for unstimulated WKY monocytes on the same EC. Although monocyte preparation procedures were minimized to prevent activation, the presence of ED-2 positive cells and the expression of VLA-4 antigen may indicate that some activation did occur. In addition, incubation of monocytes on EC monolayers for $30 \mathrm{~min}$ as is typical for adhesion assays is a suitable length of time for activation to occur. It is interesting to note that actually lower levels of CD11b/CD18 were expressed by leukocytes from stimulated hypertensive rats (1).

The comparison of monocyte adhesion to cerebromicrovascular EC from normotensive (WKY, SD) and SHR provides a unique opportunity to examine the contribution of hypertension to pathogenic mechanisms, which may affect the vascular endothelium. Despite the fact that no significant variations were observed in the quantitative dependence on any particular adhesion pathway utilized by SHR or WKY EC (antibody-blocking experiments) other differences could exist in vivo, where endothelium is subjected to shear stress and factors other than just cytokines. Another in vivo difference not addressed in vitro is related to the finding of substantially greater numbers of monocytes in SHR, which would increase the number of cell interaction events (25-27). Additional studies may identify other mechanisms through which factors such as hypertension contribute to the initiation of vascular injury and contribute to pathological changes seen in stroke and reperfusion injury.

In summary, monocytes and ECs from hypertensive animals show a greater capacity for upregulation of adhesive interactions in response to several agonists than similar cells from normotensive animals. Enhanced monocyte interaction with endothelium in segments of the vasculature may signify the ease at which overall disturbances of endothelium can occur. This increased sensitivity of SHR EC may represent a mechanism by which hypertension acts as a predisposing factor for the development of thrombotic vascular disease $(13,15,26)$.

This work was supported in part by National Institute of Neurological Disorders and Stroke Grant NS-28225.

Address for reprint requests: R. M. McCarron, Stroke Branch, NINDS, NIH, Bldg. 36, Rm. 4D-04, Bethesda, MD 20892.

Received 13 April 1994; accepted in final form 22 July 1994.

\section{REFERENCES}

1. Amdt, H., C. W. Smith, and D. N. Granger. Leukocyteendothelial cell adhesion in spontaneously hypertensive and normotensive rats. Hypertension Dallas 21: 667-673, 1993.

2. Bath, P. M. W., R. F. G. Booth, and D. G. Hassall. Monocytelymphocyte discrimination in a new microtitre-based adhesion assay. J. Immunol. Methods 118: 59-65, 1989.

3. Beekhuizen, H., A. J. Corsel-van Tilburg, I. Blokland, and R. van Furth. Characterization of the adherence of human monocytes to cytokine-stimulated human macrovascular endothelial cells. Immunology 74: 661-669, 1991.

4. Beekhuizen, H., and R. van Furth. Monocyte adherence to human vascular endothelium. J. Leukocyte Biol. 54: 363-378, 1993.

5. Bevilacqua, M. P, and R. M. Nelson. Selectins. J. Clin. Invest. 91: 379--387, 1993.

6. Carlos, T., N. Kovach, B. Schwartz, M. Rosa, B. Newman, E. Wayner, C. Benjamin, L. Osborn, R. Lobb, and J. Harlan. Human monocytes bind to two cytokine-induced adhesive ligands on cultured human endothelial cells: endothelialleukocyte adhesion molecule-1 and vascular cell adhesion molecule-1. Blood 77: 2266-2271, 1991.

7. Carlos, T. M., B. R. Schwartz, N. L. Kovach, E. Yee, M. Rosso, L. Osborn, G. Chi-Rosso, B. Newman, R. Lobb, and J. M. Harlan. Vascular cell adhesion molecule-1 (VCAM-1) 
mediates lymphocyte adherence to cytokine-activated cultured human endothelial cells. Blood 76: 965-970, 1990.

8. Cavender, D. E. Interactions between endothelial cells and the cells of the immune system. Int. Rev. Exp. Pathol. 32: 57-94, 1991.

9. Clark, W. M., K. P. Madden, R. Rothlein, and J. A. Zivin. Reduction of central nervous system ischemic injury in rabbits using leukocyte adhesion antibody treatment. Stroke 22: 877$883,1991$.

10. Cybulsky, M. I., and M. A. Gimbrone, Jr. Endothelial expression of a mononuclear leukocyte adhesion molecule during atherogenesis. Science Wash. DC 251: 788-791, 1991.

11. Dustin, M. L., and T. A. Springer. Lymphocyte functionassociated antigen-1 (LFA-1) interaction with intercellular adhesion molecule-1 (ICAM-1) is one of at least three mechanisms for lymphocyte adhesion to cultured endothelial cells. J. Cell Biol. 107: 321-331, 1988.

12. Fabry, Z., M. M. Waldschmidt, D. Hendrickson, J. Keiner, L. Love-Homan, F. Takei, and M. N. Hart. Adhesion molecules on murine brain microvascular endothelial cells: expression and regulation of ICAM-1 and Lgp 55. J. Neuroimmunol. 36: $1-11,1992$.

13. Hallenbeck, J. M., A. J. Dutka, P. M. Kochanek. A. Siren, G. H. Pezeshkpour, and G. Feuerstein. Stroke risk factors prepare rat brainstem tissues for modified local Shwartzman reaction. Stroke 19: 863-869, 1988.

14. Joris, I., T. Zand, J. J. Nannari, F. J. Krolikowski, and G. Majno. Studies on the pathogenesis of atherosclerosis. I. Adhesion and emigration of mononuclear cells in the aorta of hypercholesterolemic rats. Am. J. Pathol. 113: 341-358, 1983.

15. Liu, Y., D. M. Jacobowitz, F. Barone, R. McCarron, M. Spatz, G. Feuerstein, J. M. Hallenbeck, and A.-L. Siren. Quantitation of perivascular monocytes and macrophages around cerebral blood vessels of hypertensitive and aged rats. J. Cereb. Blood Flow Metab. 14: 348-352, 1994

16. McCarron, R. M., L. Wang, M. K. Racke, D. E. McFarlin, and $M$. Spatz. Cytokine-regulated adhesion between encephalitogenic T lymphocytes and cerebrovascular endothelial cells. $J$. Neuroimmunol. 43: 23-30, 1993.

17. McCarron, R. M., L. Wang, A.-L. Sirén, M. Spatz, and J. M. Hallenbeck. Adhesion molecules on normotensive and hypertensive rat brain endothelial cells. Proc. Soc. Exp. Biol. Med. 205: 257-262, 1994.

18. McCarron, R. M., L. Wang, D. B. Stanimirovic, and M. Spatz. Endothelin induction of adhesion molecule expression on human brain microvascular endothelial cells. Neurosci. Lett. 156: 31-34, 1993.

19. Meerschaert, J., and M. B. Furie. Monocytes use either CD11/CD18 or VLA-4 to migrate across human endothelium in vitro. J. Immunol. 152: 1915-1926, 1994.

20. Mileski, W. J., R. K. Winn, N. B. Vedder, T. H. Pohlman, J. M. Harlan, and C. L. Rice. Inhibition of CD18-dependent neutrophil adherence reduces organ injury after hemorrhagic shock in primates. Surgery 108: 206-212, 1990.

21. Osborn, L., C. Hession, R. Tizard, C. Vassallo, S. Luhowskyj, G. Chi-Rosso, and R. Lobb. Direct expression cloning of vascular cell adhesion molecule 1 , a cytokine-induced endothelial protein that binds to lymphocytes. Cell 59: 1203-1211, 1989.

22. Pober, J. S. Cytokine-mediated activation of vascular endothelium. Physiology and pathology. Am. J. Pathol. 133: 426-433, 1988.

23. Pober, J. S., and R. S. Cotran. The role of endothelial cells in inflammation. Transplantation 50: 537-544, 1990

24. Poston, R. N., D. O. Haskard, J. R. Coucher, Jr., N. P. Gall, and R. R. Johnson-Tidey. Expression of intercellular adhesion molecule-1 in atherosclerotic plaque. Am. J. Pathol. 140: 665$673,1992$.

25. Schmid-Schönbein, G. W., D. Seiffge, F. A. DeLano, K. Shen, and B. W. Zweifach. Leukocyte counts and activation in spontaneously hypertensive and normotensive rats. Hypertension Dallas 17: 323-330, 1991.

26. Siren, A.-L., E. Heldman, D. A. Doron, P. G. Lysko, T. L. Yue, Y. Liu, G. Feuerstein, and J. M. Hallenbeck. Release of proinflammatory and prothrombic mediators in the brain and peripheral circulation in spontaneously hypertensive and normotensive Wistar-Kyoto rats. Stroke 23: 1643-1651, 1992.

27. Siren, A.-L., R. M. McCarron, Y. Liu, E. Heldman, M. Spatz, G. Feuerstein, and J. M. Hallenbeck. Perivascular macrophage signaling of endothelium via cytokines: mechanism by which stroke risk factors operate to increase stroke likelihood. In: Pharmacology of Cerebral Ischemia, edited by J. Krieglstein and H. Oberpichler-Schwenk. Stuttgart, Germany: Wissenschaftliche, 1992, p. 435-447.

28. Sobel, R. A., M. E. Mitchell, and G. Fondren. Intercellular adhesion molecule-1 (ICAM-1) in cellular immune reactions in the human central nervous system. Am J. Pathol. 136: 1309-1316, 1990.

29. Spatz, M., J. Bembry, R. F. Dodson, H. Hervonen, and M. R. Murray. Endothelial cell culture derived from isolated cerebral microvessels. Brain Res. 191: 577-582, 1980.

30. Tanaka, M., and R. M. McCarron. The inhibitory effect of tumor necrosis factor and interleukin-1 on Ia induction by interferon- $\gamma$ on endothelial cells from murine central nervous system microvessels. J. Neuroimmunol. 27: 209-215, 1990.

31. Van Furth, R., and W. Sluiter. Distribution of blood monocytes between a marginating and a circulating pool. J. Exp. Med. 163: 474-479, 1986.

32. Winn, R. K., and J. M. Harlan. CD-18-independent neutrophil and mononuclear leukocyte emigration into the peritoneum of rabbits. J. Clin. Invest. 92: 1168-1173, 1993.

33. Wuthrich, R. P., A. M. Jevnikar, F. Takei, L. H. Glimcher, and V. E. Kelly. Intercellular adhesion molecule-1 (ICAM-1) expression is upregulated in autoimmune murine lupus nephritis. Am. J. Pathol. 136: 441-450, 1990. 